\title{
COMPORTAMENTO DE ÉGUAS APÓS A INSERÇÃO DE DISPOSITIVO INTRAVAGINAL IMPREGNADO COM PROGESTERONA
}

\author{
Antonio Campanha Martinez ${ }^{8}$ \\ Marcos Henrique Alcantara Colli ${ }^{9}$ \\ Rafael Silveira Carvalho ${ }^{10}$ \\ Joaquim Oliveira Moleirinho ${ }^{11}$ \\ Maycon Araujo Ruivo ${ }^{12}$ \\ Adalgiza Pinto Neto ${ }^{13}$ \\ Welber Daniel Lopes ${ }^{14}$
}

MARTINEZ, A. C.; COLli, M. H. A.; CARVAlHO, R. S.; MOLEIRINHO, J. O.; RUIVO, M. A.; PINTO NETO, A.; LOPES, W. D. Comportamento de éguas após a inserção de dispositivo intravaginal impregnado com progesterona. Arq. Ciênc. Vet. Zool. UNIPAR, Umuarama, v. 19, n. 3, p. 143-146, jul./set. 2016.

RESUMO: Considerando a escassez de estudos sobre o temperamento ou reatividade de equinos, objetivou-se com esse estudo avaliar o comportamento de éguas após a inserção do implante intravaginal de progesterona, para utilização em bovinos, por meio da observação de comportamentos individuais e coletivos. Para tanto, utilizou-se sete éguas que receberam dispositivo intravaginal impregnado com progesterona e agente luteolítico. Após a colocação dos implantes e soltura das éguas, se iniciou a observação (cinco observadores sem contato entre eles) de seus comportamentos até o tempo de 120 minutos, seguindo o modelo proposto por Heleski et al. (2002). Os resultados indicaram que durante os 120 minutos de observação todas as éguas demonstraram estar alertas, pelo menos em uma observação; que o ato de comer foi observado em todos os animais durante o período estudado e que o ato de urinar e defecar foram observados, em aproximadamente, 70 e $60 \%$, respectivamente, dos comportamentos avaliados. Comportamentos prováveis da indicação de dor e/ou desconforto, como bater da cauda, mímica de urinar, eversão do clitóris e urinando foram observados até 65 minutos, sendo que os três primeiros cessaram aos 55 minutos. De todos os comportamentos analisados, nenhum deles foi visualizado após 65 minutos de observação. Concluiu-se que a inserção do dispositivo intravaginal impregnado com progesterona não afeta negativamente o comportamento de éguas, podendo ser utilizado sem comprometer o bem-estar desses animais.

PALAVRAS-CHAVE: Equino. Temperamento. Reatividade. Sincronização de estro.

\section{BEHAVIOR OF MARES AFTER INSERTION OF INTRAVAGINAL PROGESTERONE DEVICE}

\begin{abstract}
Considering the scarcity of studies on the behavior or reactivity of horses, this study aimed to assess the behavior of mares after insertion of intravaginal progesterone implant for use in cattle, through the observation of individual and collective behavior. In order to do so, seven mares received intravaginal devices impregnated with progesterone and luteolitic agent. After the implant was placed, the mares were released, and their behavior was observed (five observers with no contact between them) for a period of 120 minutes, following the model proposed by Heleski et al. (2002). The results indicated that during the 120-minute period, all mares were alert, in at least one observation; that all animals ate during the study period, and urination and defecation were observed in approximately 70 and $60 \%$, respectively, of the assessed behavior. Behaviors that were likely an indication of pain and/or discomfort, like hitting the tail, miming to urinate, eversion of the clitoris and urinating were observed in up to 65 minutes, with the first three symptoms ceasing after 55 minutes. None of the analyzed behaviors were viewed after 65 minutes of observation. It can be concluded that the insertion of the intravaginal device impregnated with progesterone does not adversely affect the behavior of mares, and therefore, it can be used without compromising the well-being of the animals.
\end{abstract}

KEY WORDS: Equine. Behavior. Reactivity. Synchronization of estrus.

\section{COMPORTAMIENTO EN YEGUAS DESPUÉS DE LA INSERCIÓN DEL DISPOSITIVO INTRAVAGINAL IMPREGNADO CON PROGESTERONA}

RESUMEN: Teniendo en cuenta la escasez de estudios sobre el temperamento o la reactividad de equinos, este estudio buscó evaluar el comportamiento de yeguas después de la inserción del implante de progesterona intravaginal, para su uso en ganado, a través de observación de conductas individuales y colectivas. Para ello, se utilizó siete yeguas que recibieron dispositivo intravaginal impregnado con progesterona y agente luteolítico. Después de los implantes y la liberación de las

DOI: https://doi.org/10.25110/arqvet.v19i3.2016.6086

${ }^{8}$ Docente. Programa de Pós-Graduação em Saúde e Produção Animal Sustentável. Universidade Estadual de Maringá. Campus Umuarama. Correspondência ao autor: acmartinez@uem.br

${ }^{9}$ Mestrando. FMVZ- USP

${ }^{10}$ Mestrando. FMVZ- Unesp-Botucatu

${ }^{11}$ Médico Veterinário. Agropecuária Luzipar

${ }^{12}$ Mestrando. Programa de Pós-Graduação em Saúde e Produção Animal Sustentável. Universidade Estadual de Maringá. Campus Umuarama

${ }^{13}$ Docente do Curso de Medicina Veterinária. Campus Realeza-UFFS.

${ }^{14}$ Docente - Universidade Federal de Goiás - Goiânia. 
yeguas, comenzó la observación (cinco observadores sin contacto entre ellos) de sus comportamientos hasta el tiempo de 120 minutos, siguiendo el modelo propuesto por Heleski et al. (2002). Los resultados indicaron que durante los 120 minutos de observación todas las yeguas demostraron estar alertas, por lo menos en una observación; el acto de comer se observó en todos los animales durante el periodo de estudio y que el acto de la micción y la defecación, en aproximadamente, 70 y $60 \%$, respectivamente, de los comportamientos evaluados. Comportamientos probables de indicación de dolor y/o malestar, como golpear la cola, la mímica de orinar, eversión del clítoris y orinando, observados hasta 65 minutos, siendo que los tres primeros cesaron después de 55 minutos. De todos los comportamientos analizados, ninguno de ellos se visualizó después de la observación de 65 minutos. Se concluyó que la inserción del dispositivo intravaginal impregnado con progesterona no afecta negativamente el comportamiento de yeguas, que puede ser utilizado sin comprometer el bienestar de esos animales.

PALABRAS CLAVE: Equino. Temperamento. Reactividad. Sincronización de estro.

\section{Introdução}

Poucos e recentes estudos têm sido realizados para avaliar o temperamento ou reatividade dos equinos (ANDERSON et al., 1999; JEZIERSKI; JAWORSKI; GO'RECKA, 1999). Na égua, o comportamento sexual está envolvido no contexto dos grupos familiares, formados tipicamente por éguas adultas e seus potros, e um ou mais garanhões (KASEDA et al., 1995; AURICH, 2011).

$\mathrm{Na}$ maioria das espécies unguladas, como os equinos, machos e fêmeas ficam juntos apenas na estação de monta, e separados durante o resto do ano, sendo que o desenvolvimento de comportamentos não usuais é provavelmente significante para a mantença de longas relações entre éguas e garanhões (CROWELL-DAVIS, 2007).

A hierarquia dentro de um grupo familiar é determinada por vários fatores, como tamanho, idade e tempo de residência dentro do grupo, que podem ser preditivos de alta posição (RHO; SRYGLEY; CHOEM, 2004).

A maioria das éguas apresenta comportamento reprodutivo estacional, não sendo férteis durante todo o ano. A sazonalidade reprodutiva em equinos refere-se à mudanças morfológicas e fisiológicas dos órgãos reprodutivos, primariamente devido à mudanças na relação diária de luminosidade e escuridão durante do ano (BERGFELT, 2009), que associados, estimularam a busca por conhecimentos relativos aos mecanismos da sazonalidade reprodutiva e ao desenvolvimento de métodos para uma indução antecipada da estação de monta (NAGY; GUILLAUME; DAELS, 2000; AURICH, 2011).

Dentro desse contexto, o uso de progesterona exógena, em dispositivos intravaginais ou subcutâneos auriculares, associado a outras drogas, possibilita a realização de protocolos de sincronização de estro e ovulação, permitindo que éguas produzam seus potros o mais rápido possível do ano hípico (SCHUTZER, 2012). Existem muitas razões pelas quais se faz necessário a busca de novos métodos para que as éguas possam entrar em estro e obter um potro o mais próximo possível do início do ano hípico, sendo a principal, a adequação do nascimento dos produtos à data oficial imposta para cavalos de performance (DONADEU; WATSON, 2007). Nesse contexto, o Brasil é referência em estudo e utilização de biotécnicas de reprodução assistida nesses animais (OLIVEIRA FILHO et al., 2012).

Para que obtenha a máxima eficiência com o uso das técnicas de reprodução assistida nas espécies animais, deve-se conhecer além da fisiologia reprodutiva, o comportamento sexual desses animais, possibilitando a potencialização dos processos reprodutivos, por meio do uso de tecnologias e procedimentos específicos no campo e/ou laboratório
(PEÑA-ALFARO, 2008).

Dessa forma, objetiva-se com esse estudo avaliar o comportamento de éguas após a inserção do implante intravaginal para utilização em bovinos (CIDR ${ }^{\circledR}$, Zoetis, São Paulo - Brasil), por meio da avaliação de comportamentos individuais e coletivos.

\section{Material e Metódos}

O experimento foi realizado no Laboratório de Criação e Reprodução Animal da Universidade Estadual de Maringá, Campus de Umuarama (23 $47^{\prime} 55 \mathrm{~S}, 53^{\circ} 18^{\prime} 48$ O), Estado do Paraná, do final de outubro ao início de novembro de 2013. Para tanto, foram utilizadas sete éguas não gestantes, sem potro ao pé, sem raça definida, em idade reprodutiva, mantidas em regime de pastejo natural e com livre acesso à água.

As éguas foram contidas em tronco de contenção específico e todas receberam o mesmo tratamento. Após a lavagem do períneo e lábios vulvares, colocou-se o dispositivo intravaginal impregnado com progesterona ${ }^{8}$, de uso específico para bovinos, e aplicou-se um agente luteolítico, contendo $225 \mathrm{mg}$ de D-cloprostenol ${ }^{9}$.

Imediatamente após a soltura dos animais do tronco de contenção foi iniciada a observação do comportamento desses animais. No momento da soltura considerou-se o tempo zero. Desde então, cinco observadores, sem contato entre eles, iniciaram a observação do comportamento dos animais, até o tempo de 120 minutos. Cada observador munido de cronômetro e planilha contendo os comportamentos a serem observados (mantendo-se alerta, mantendo-se relaxada, deitando esternalmente, rolando, comendo, andando em círculo, comportamento agressivo, comportamento amigável, batendo cauda, urinando, mímica de urinar, defecando, eversão, coughing e relaxando), preencheu a planilha no momento exato da constatação de comportamento das éguas, de maneira semelhante ao modelo proposto por Heleski et al. (2002).

Os dados coletados foram organizados em uma planilha única e apresentados por meio de estatística descritiva, utilizando-se o Microsoft Excel.

\section{Resultados e Discussão}

A Figura 1 apresenta a prevalência de comportamentos exibidos pelos animais experimentais até os $120 \mathrm{mi}-$ nutos após colocação de dispositivo intravaginal impregnado com progesterona.

${ }^{8} \mathrm{CIDR}^{\circledR}$, Zoetis, São Paulo - Brasil

${ }^{9}$ Veteglan ${ }^{\circledR}$, Hertape-Calier, Juatuba-MG, Brasil 
Figura 1: Prevalência de comportamentos exibidos por éguas até 120 minutos após colocação de dispositivo intravaginal impregnado com progesterona.

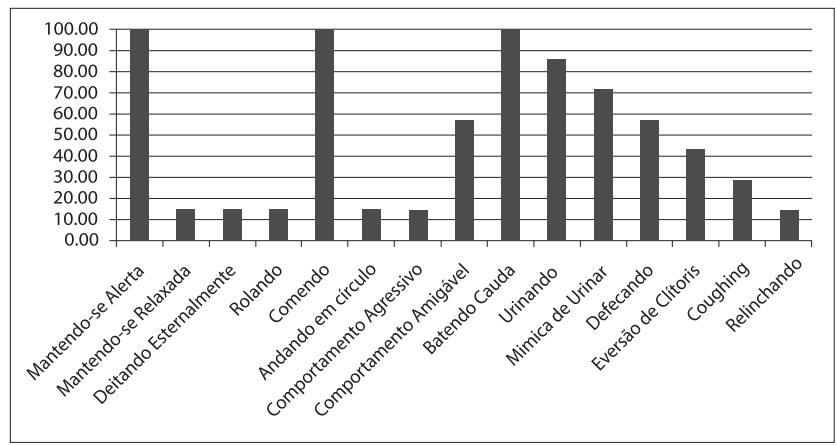

Durante os 120 minutos de observação, todas as éguas estudadas demonstraram estar alertas, pelo menos em uma observação (Figura 1). Essa observação se justifica, uma vez que os equinos podem ser considerados animais predados (SWANN, 2006).

De maneira semelhante, o ato de comer foi observado em todos os animais durante o período estudado (Figura 1). Budianski (1997) relatou que os equinos, sendo animais de rebanho, passam mais de $50 \%$ do dia pastando. Adicionalmente, Short (1998) relatou que o consumo alimentar, e o ato de urinar e defecar contribui para melhorar a tolerância à dor. $\mathrm{O}$ ato de urinar e defecar foram observados, em aproximadamente, 70 e $60 \%$, respectivamente, dos comportamentos avaliados (Figura 1).

Comportamentos prováveis da indicação de dor e/ ou incômodo vaginal (CHRISTOFFERSEN; LEHN-JENSEN; BØGH, 2007), como bater da cauda, mímica de urinar, eversão do clitóris e urinando foram observados até $65 \mathrm{mi}$ nutos, sendo que os três primeiros cessaram aos 55 minutos (Quadro 1).

Em estudo semelhante, utilizando vacas Nelore, Rueda et al. (2011) observaram que a inserção de um dispositivo intravaginal, semelhante ao utilizado nesse estudo, não alterou o comportamento e o bem-estar desses animais. Nesse estudo, comportamentos indicadores de incômodo, como apresentados no Quadro 1 (estado de alerta, batimento de cauda e mímica de urinar) cessaram aos 55 minutos, sugerindo, baseando-se nos relatos de Rueda et al. (2011), que o dispositivo utilizado não alterou o bem-estar das éguas estudadas.

De todos os comportamentos analisados (Quadro 1), nenhum deles foi visualizado após 65 minutos de observação. Outros comportamentos descritos por Heleski et al. (2002), embora considerados, não foram apresentados pelos animais estudados.

Quadro 1: Comportamentos exibidos por éguas até 120 minutos após colocação de dispositivo intravaginal impregnado com progesterona.

\begin{tabular}{|c|c|c|c|c|c|c|c|c|c|c|c|c|c|c|c|c|}
\hline \multirow[b]{2}{*}{ Comportamento } & \multicolumn{15}{|c|}{ Minutos de observação } & \multirow{17}{*}{ 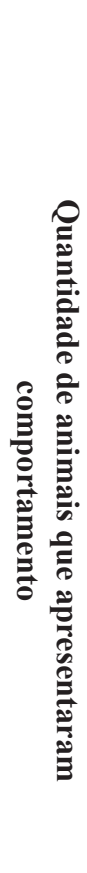 } \\
\hline & 0 & 5 & 10 & 15 & 20 & 25 & 30 & 35 & 40 & 45 & 50 & 55 & 60 & 65 & 70 & \\
\hline Mantendo-se Alerta & - & - & 1 & 1 & 1 & 1 & 1 & 2 & - & 1 & - & 1 & - & - & - & \\
\hline Mantendo-se Relaxada & - & - & - & - & - & - & - & - & - & - & 1 & - & - & - & - & \\
\hline Deitando Esternalmente & - & - & - & - & - & - & - & - & - & - & - & 1 & - & - & - & \\
\hline Rolando & - & - & - & - & - & - & - & - & - & - & - & - & - & - & - & \\
\hline Comendo & - & - & 5 & 4 & 4 & - & 4 & - & - & - & - & - & - & - & - & \\
\hline Andando em círculo & - & - & - & - & - & 1 & - & - & - & - & - & - & - & - & - & \\
\hline Comportamento Agressivo & - & - & - & - & - & - & - & - & 1 & - & - & - & - & - & - & \\
\hline Comportamento Amigável & - & - & - & - & 2 & - & - & 5 & - & - & 1 & - & - & - & - & \\
\hline Batendo Cauda & 5 & - & 4 & 6 & - & - & 1 & - & - & - & 1 & - & - & - & - & \\
\hline Urinando & - & - & 3 & 2 & 1 & 1 & - & 2 & - & 1 & 1 & 2 & 1 & 2 & - & \\
\hline Mímica de Urinar & - & - & 1 & 1 & - & - & - & - & 2 & - & 1 & - & - & - & - & \\
\hline Defecando & - & - & - & 1 & 2 & 2 & 1 & 1 & 1 & 2 & 1 & 1 & - & - & - & \\
\hline Eversão de Clítoris & - & - & 1 & - & - & - & - & - & - & 1 & - & 1 & - & - & - & \\
\hline Coughing & - & - & - & - & - & - & 1 & 1 & - & - & - & - & - & - & - & \\
\hline Relinchando & - & - & - & - & 1 & - & - & - & 1 & - & - & - & - & - & - & \\
\hline
\end{tabular}

\section{Conclusões}

Nas condições desse estudo, observou-se que a inserção do dispositivo intravaginal impregnado com progesterona não afeta negativamente o comportamento de éguas. Dessa forma, os resultados relatados subsidiam o uso desse dispositivo em éguas, sem comprometimento do comportamento e/ou bem-estar desses animais, na rotina da sincronização do estro e ovulação de éguas.

\section{Referências}

ANDERSON, M. K. et al. Behavioral assessment of horses in therapeutic riding programs. Applied Animal Behavior Science, v. 63, p. 1-24, 1999.

AURICH, C. Reproductive cycles of horses. Animal Reproduction Science, v. 124, p. 220-228, 2011.

BERGFELT, D. R. Anatomy and physiology of the mare. In: SAMPER, J. C. 2. ed. Equine breeding management 
and artificial insemination. Missouri:Saunders Elsevier, 113-131p. 2009.

BUDIANSKI, S. The nature of horses. The freepress: New York. 1997.

CHRISTOFFERSEN, M.; LEHN-JENSEN, H.; BØGH, I. B. Referred vaginal pain: cause of hypersensitivity and performance problems in mares? A clinical case study. Journal of Equine Veterinary Science, v. 27, p. 32-36, 2007.

CROWELL-DAVIS, S. L. Sexual behaviorof mares. Hormones and Behavior, v. 52, p. 12-17, 2007.

DONADEU, F. X.; WATSON, E. D. Seasonal changes in ovarian activity: lessons learnt from the horse. Animal Reproduction Science, v. 100, p. 225-242, 2007.

HELESKI, C. R. et al. Influence of housing on weanling horse behaviorand subsequente welfare. Applied Animal Behavior Science, v. 78, p. 291-302, 2002.

JEZIERSKI, T.; JAWORSKI, Z.; GO'RECKA, A. Effects of handling on behavioran heart rate in Konik horses: comparasion of stable and forester a red young stock. Applied Animal Behavior Science, v. 62, p. 1-11, 1999.

KASEDA, Y.; KHALIL, A.M.; OGAWA, H. Harem stability and reproductive success of Misakiferal mares. Equine Veterinary Journal, v. 27, p. 368-72, 1995.

NAGY, P.; GUILLAUME, D.; DAELS, P. Seasonality in mares. Animal Reproduction Science, v. 60/61, p. $245-$ $262,2000$.

OLIVEIRA FILHO, L. R. et al. Efeito do implante intravaginal de progesterona sobre a ciclicidade de éguas em anestro da raça Quarto de Milha. Nucleus Animalium, v. 4, p. 113-119, 2012.

PENÃ-ALFARO, C. E. Reprodução de equinos e asininos. Apontamentos. Programa de Pós-graduação em Medicina Veterinária da UFCG, Patos, 2008.30p.

RHO, J. R.; SRYGLEY, R. B.; CHOEM, J. C. Behavioral ecology of the Jeju pony (Equus caballus): Effects of maternal age, maternal dominance hierarchy and foal age on mare agression. Ecological Research, 19: 55-63, 2004.

RUEDA, P. M. et al. Differences behavioural in Nelore cows before and after use of intra vaginal device of progesterone. IN: ISAE - 45th Congress of International Society of Applied Ethology, p.158, 2011, Indianapolis. Anais... Indianapolis: CD-ROM.

SHORT, C. E. Fundamentals of pain perception in animals. Applied Animal Behaviour Science, v. 59, p. 125-133, 1998. progesterona intra-vaginal e acetato de deslorelina em éguas acíclicas associados ou não a luz artificial para o controle da sazonalidade reprodutiva. Botucatu, 2012. 62p. Dissertação (Mestrado). Faculdade de Medicina Veterinária e Zootecnia, Universidade Estadual Paulista.

SWANN, W. J. Improving the welfare of working equine animals in developing countries. Applied Animal Behaviour Science, v. 100, p. 148-151, 2006.

\section{SCHUTZER, C. G. C. Utilização do implante de}

